\title{
Polymer model with Epigenetic Recoloring Reveals a Pathway for the de novo Establishment and 3D Organization of Chromatin Domains
}

\author{
D. Michieletto, ${ }^{1}$ E. Orlandini, ${ }^{2}$ and D. Marenduzzo ${ }^{1}$ \\ ${ }^{1}$ SUPA, School of Physics and Astronomy, University of Edinburgh, \\ Peter Guthrie Tait Road, Edinburgh EH9 3FD, United Kingdom \\ ${ }^{2}$ Dipartimento di Fisica e Astronomia and Sezione INFN, \\ Università di Padova, Via Marzolo 8, Padova 35131, Italy
}

(Received 15 June 2016; revised manuscript received 5 August 2016; published 9 December 2016)

One of the most important problems in development is how epigenetic domains can first be established, and then maintained, within cells. To address this question, we propose a framework that couples threedimensional chromatin folding dynamics to a "recoloring" process modeling the writing of epigenetic marks. Because many intrachromatin interactions are mediated by bridging proteins, we consider a "two-state" model with self-attractive interactions between two epigenetic marks that are alike (either active or inactive). This model displays a first-order-like transition between a swollen, epigenetically disordered phase and a compact, epigenetically coherent chromatin globule. If the self-attraction strength exceeds a threshold, the chromatin dynamics becomes glassy, and the corresponding interaction network freezes. By modifying the epigenetic read-write process according to more biologically inspired assumptions, our polymer model with recoloring recapitulates the ultrasensitive response of epigenetic switches to perturbations and accounts for long-lived multidomain conformations, strikingly similar to the topologically associating domains observed in eukaryotic chromosomes.

DOI: 10.1103/PhysRevX.6.041047

\section{INTRODUCTION}

The word "epigenetics" refers to heritable changes in gene expression that occur without alterations of the underlying DNA sequence [1,2]. By now, it is well established that such changes often arise through biochemical modifications occurring on histone proteins while they are bound to eukaryotic DNA to form nucleosomes, the building blocks of the chromatin fiber [1]. These modifications, or "epigenetic marks," are currently thought of as forming a "histone code" [3], which ultimately regulates expression [4].

It is clear that this histone code has to be established de novo during cell development and inherited after each cell cycle through major genetic events such as replication, mitosis, or cell division [5]. A fundamental question in cell biology and biophysics is, therefore, how certain epigenetic patterns are established and what mechanism can make them heritable. One striking example of epigenetic imprinting is the "X-chromosome inactivation," which refers to the silencing of one of the two $X$ chromosomes within the nucleus of mammalian female cells - this is crucial to avoid overexpression of the genes

Published by the American Physical Society under the terms of the Creative Commons Attribution 3.0 License. Further distribution of this work must maintain attribution to the author(s) and the published article's title, journal citation, and DOI.
Subject Areas: Biological Physics, Soft Matter, Statistical Physics in the $\mathrm{X}$ chromosomes, which would ultimately be fatal for the cell. While the choice of which chromosome should be inactivated is stochastic within embryonic stem cells, it is faithfully inherited in differentiated cells [6]. The inactivation process is achieved, in practice, through the spreading of repressive histone modifications, which turn the chromosome into a transcriptionally silenced Barr body [7-9]. This is an example of an "epigenetic switch," a term that generically refers to the up or down regulation of specific genes in response to, e.g., seasonal changes [10-12], dietary restrictions [13], aging [14], or parental imprinting [15].

Although one of the current paradigms of the field is that the epigenetic landscape and 3D genome folding are intimately related [16-24], most of the existing biophysical studies incorporating epigenetic dynamics have focused on one-dimensional (1D) or mean-field models [25-34]. While these models can successfully explain some aspects of the establishment, spreading, and stability of epigenetic marks, they cannot fully capture the underlying threedimensional (3D) dynamic organization of the chromatin. However, the dynamical 3D folding may be a key aspect to consider: For instance, repressive epigenetic modifications are thought to correlate with chromatin compaction $[1,29]$; therefore, it is clear that there must be a strong feedback between the self-regulated organization of epigenetic marks and the 3D folding of chromatin. In light of this, here we propose a polymer model of epigenetic switches, which 
directly couples the 3D dynamics of chromatin folding to the $1 \mathrm{D}$ dynamics of epigenetics spreading.

More specifically, we start from the observation that there are enzymes that can either "read" or "write" epigenetic marks (Fig. 1). The "readers" are multivalent proteins [17] that bridge chromatin segments bearing the same histone marks. The "writers" are enzymes that are responsible for the establishment and propagation of a specific epigenetic mark, perhaps while performing facilitated diffusion along chromatin [35]. There is evidence that writers of a given mark are recruited by readers of that same mark $[12,25,26,28,29,36-38]$, thereby creating a positivefeedback loop that can sustain epigenetic memory [26]. For example, a region that is actively transcribed by an RNA polymerase is rich in active epigenetic marks (such as the H3K4-methylated marks) [36,39]: The polymerase in this

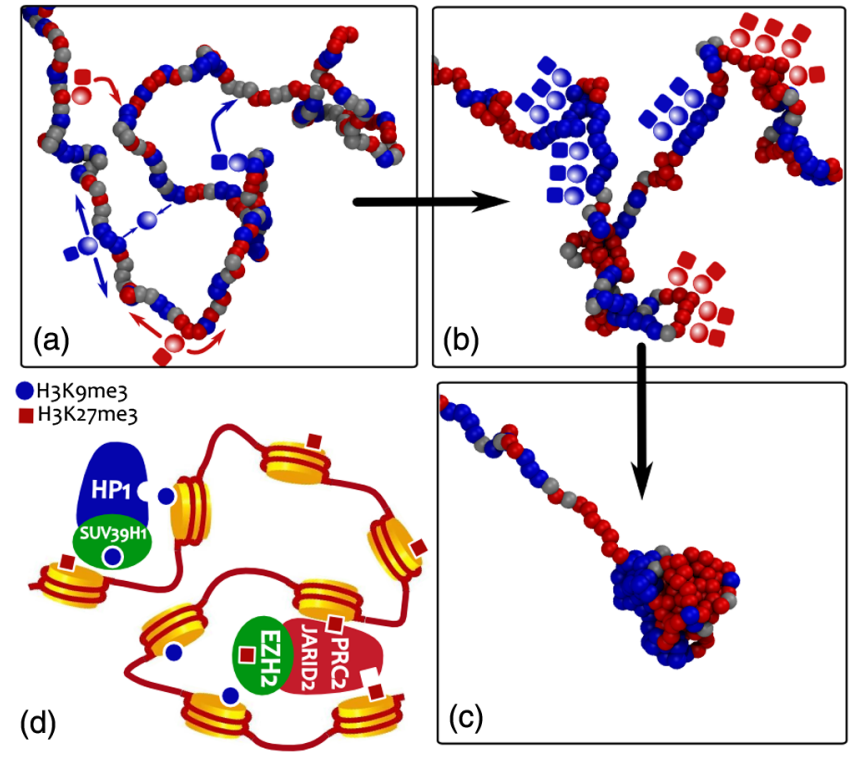

FIG. 1. A 3D polymer model with "recoloring" for the propagation of epigenetic marks. (a)-(c) Multivalent binding proteins, or readers (shaded spheres), bind to specific histone modifications and bridge between similarly marked segments (distinguished here via their "color"). Histone-modifying enzymes, or writers (solid squares), are assumed here to be chaperoned by the bridge proteins. The writing (or recoloring) activity is a consequence of $3 \mathrm{D}$ contiguity (perhaps through facilitated diffusion [35]), which is modeled here as a Potts-like interaction between spatially proximate monomers [41] (a). The positive-feedback mechanism and competition between different epigenetic marks results in a regulated spreading of the modifications (b), which, in turn, drives the overall folding of the polymer (c). A sketch of a biological reading-writing machinery is shown in (d). Heterochromatin binding protein HP1 is known to recruit methyltransferase proteins (e.g., SUV39H1), which, in turn, trimethylates lysine 9 on histone 3 (H3K9me3) $[29,39,42]$. Similarly, the Polycomb Repressive Complex (PRC2) is known to comprise the histone H3 Lys 27 (H3K27) methyltransferase enzyme EZH2 $[12,39,43]$ while binding the same mark through the interaction with JARID2 $[43,44]$. example is a reader that recruits the writer Set1/2 [39,40]. Likewise, the de novo formation of centromeres in human nuclei occurs through the creation of the centromerespecific nucleosome CENP-A (a modified histone, which can thus be viewed as an epigenetic mark) via the concerted action of the chaperone protein HJURP (the writer) and the Mis18 complex (the reader) [38]. Other examples of this read-write mechanism are shown in Fig. 1. This mechanism creates a route through which epigenetic marks can spread to spatially proximate regions on the chromatin, and it is responsible for the coupling between the $3 \mathrm{D}$ folding and 1D epigenetic dynamics, addressed for the first time in this work.

Here, we find that, for the simplest case of only two epigenetic states that symmetrically compete with each other (e.g., corresponding to "active" or "inactive" chromatin [1]), our model predicts a first-order-like phase transition between a swollen, epigenetically disordered phase and a collapsed, epigenetically coherent one. The first-order nature of the transition, within our model, is due to the coupling between 3D and 1D dynamics, and it is important because it allows for a bistable epigenetic switch that can retain memory of its state. When quenching the system to well below the transition point, we observe a faster 3D collapse of the model chromatin; surprisingly, this is accompanied by a slower 1D epigenetic dynamics. We call this regime a "glassy" phase, which is characterized, in 3D, by a frozen network of strong and short-ranged intrachain interactions giving rise to dynamical frustration and the observed slowing down, and, in 1D, by a large number of short epigenetic domains.

If the change from one epigenetic mark to the other requires going through an intermediate epigenetic state, we find two main results. First, a long-lived metastable mixed state (MMS), previously absent, is now observed: This is characterized by a swollen configuration of the underlying chain where all epigenetic marks coexist. Second, we find that the MMS is remarkably sensitive to external local perturbations, while the epigenetically coherent states, once established, still display robust stability against major reorganization events, such as replication. This behavior is reminiscent of the features associated with epigenetic switches and the X-chromosome inactivation.

We conclude our work by looking at the case in which the epigenetic writing is an ATP-driven, and hence a nonequilibrium, process. In this case, detailed balance is explicitly broken, and there is no thermodynamic mapping of the underlying stochastic process. This case leads to a further possible regime, characterized by the formation of a long-lived multipearl structure, where each "pearl" (or chromatin domain) is associated with a distinct epigenetic domain. This regime is qualitatively different from the glassy phase, as the domains reach a macroscopic size and a significant fraction of chain length. Finally, these self-organized structures are reminiscent of "topologically 
associating domains" (TADs), experimentally observed in chromosomal contact maps [45].

\section{MODELS AND METHODS}

We model the chromatin fiber as a semiflexible bead-andspring chain of $M$ beads of size $\sigma$ [17,46-50]. For concreteness, we consider $\sigma=3 \mathrm{kbp} \simeq 30 \mathrm{~nm}$, approximately corresponding to 15 nucleosomes-this mapping is commonly used when modeling chromatin dynamics $[46,47,50]$. To each bead, we assign a "color" $q$ representing a possible epigenetic state (mark). Here, we consider $q \in\{1,2,3\}$, i.e., three epigenetic marks such as methylated (inactive), unmarked (intermediate), and acetylated (active).

In addition to the standard effective potentials to ensure chain connectivity (through a harmonic potential between consecutive beads) and bending rigidity (through a KratkyPorod potential [51]), we consider a repulsive or attractive interaction mediated by the epigenetic marks (colors). This is described by a truncated-and-shifted Lennard-Jones potential, defined as follows:

$$
\begin{aligned}
U_{L J}^{a b}(x)= & \frac{4 \epsilon_{a b}}{\mathcal{N}}\left[\left(\frac{\sigma}{x}\right)^{12}-\left(\frac{\sigma}{x}\right)^{6}-\left(\frac{\sigma}{x_{c}^{q_{a} q_{b}}}\right)^{12}+\left(\frac{\sigma}{x_{c}^{q_{a} q_{b}}}\right)^{6}\right] \\
& \text { for } x \leq x_{c}^{q_{c} q_{b}},
\end{aligned}
$$

whereas $U_{L J}^{a b}(x)=0$ for $x>x_{c}^{q_{a} q_{b}}$. In Eq. (1), $\mathcal{N}$ is a normalization constant and the parameter $\epsilon_{a b}$ is set so that $\epsilon_{a b}=\epsilon$ for $q_{a}=q_{b}$ and $\epsilon_{a b}=k_{B} T_{L}$ otherwise. The $q$-dependent interaction cutoff $x_{c}^{q_{a} q_{b}}$ is given by $2^{1 / 6} \sigma$, to model steric repulsion, or $R_{i}>2^{1 / 6} \sigma$ to model attraction. (Here, we consider $R_{i}=1.8 \sigma$, which simultaneously ensures short-range interaction and computational efficiency.) In what follows, the cutoffs are chosen so that beads with different colors, or with color corresponding to no epigenetic marks (i.e., $q=3$ ), interact via steric repulsion, whereas beads with the same color, and corresponding to a given epigenetic mark (e.g., $q=1$ or $q=2$ ), self-attract modeling interactions mediated by a bridging protein, one of the "readers" $[1,17]$.

The time evolution of the system is obtained by coupling a 3D Brownian polymer dynamics at temperature $T_{L}$, with a recoloring Monte Carlo dynamics of the beads, which does not conserve the number of monomer types. Recoloring moves are proposed every $\tau_{\operatorname{Rec}}=10^{3} \tau_{\mathrm{Br}}$, where $\tau_{\mathrm{Br}}$ is the Brownian time associated with the dynamics of a single polymer bead, and they are realized, in practice, by attempting $M$ changes of the beads color. To compare between simulation and physical time units, a Brownian time $\tau_{\mathrm{Br}}$ is mapped here to $10 \mathrm{msec}$, corresponding to an effective nucleoplasm viscosity $\eta \simeq 150 \mathrm{cP}$. This is an intermediate and conservative value within the range that can be estimated from the literature $[47,52]$ and from a direct mapping with the experimental data of Ref. [53] (see Ref. [54], Fig. S1). With this choice, the recoloring rate is about $0.1 \mathrm{~s}^{-1}$, and a simulation runtime of $10^{6}$ Brownian times corresponds to 2.5-3 hours (see Ref. [54] for more details on the mapping). Each color change is accepted according to the standard Metropolis acceptance ratio, with effective temperature $T_{\operatorname{Rec}}$ and a Potts-like energy difference computed between beads that are spatially proximate (i.e., within distance $R_{i}$ in 3D). It is important to notice that, whenever $T_{L} \neq T_{\mathrm{Rec}}$, detailed balance of the full dynamics is broken, which may be appropriate if epigenetic spreading and writing depend on nonthermal processes (e.g., if they are ATP driven). More details on the model and values of all simulation parameters are given in Ref. [54], Fig. S1 [55].

The model we use therefore couples an Ising-like (or Potts-like) epigenetic recoloring dynamics to the threedimensional kinetics of polymer folding. In most simulations we consider, for simplicity, $T_{L}=T_{\mathrm{Rec}}$, and we start from an equilibrated chain configuration in the swollen phase (i.e., at very large $T_{L}$ ), where beads are randomly colored with uniform probability. The polymer and epigenetic dynamics is then studied by tuning the interaction parameter $\alpha=\epsilon / k_{B} T_{L}$ to values near or below the critical value $\alpha_{c}$ for which we observe the polymer collapse.

\section{RESULTS}

\section{A. The two-state model displays a first-order-like transition, which naturally explains both epigenetic memory and bistability}

For simplicity, we focus here on the case in which three states are present; however, only two of them $[q=1$ (red) and $q=2$ (blue)] are self-attractive, while the third is a neutral state that does not self-attract but can participate in coloring dynamics $(q=3$, grey). Transitions between any two of these three states are possible in this model. Because we find that the grey (unmarked) state rapidly disappears from the polymer at the advantage of the self-attractive ones, we refer to this as an effectively "two-state" model. This scenario represents the case with two competing epigenetic marks (e.g., an active acetylation mark and an inactive methylation mark), while the third state represents unmarked chromatin.

Figure 2 reports the polymer and epigenetic dynamics (starting from the swollen and randomly colored initial state), for two different values of $\alpha=\epsilon / k_{B} T_{L}$ below and above the critical point $\alpha_{c}$. The global epigenetic recoloring is captured by $N_{b}(q, t)$, the total number of beads in state $q$ at time $t$; the local epigenetic dynamics is instead represented by a "kymograph" [57], which describes the change in color of the polymer beads as time evolves (Fig. 2).

It is readily seen that above the critical point $\alpha_{c} \simeq 0.9$ (for $M=2000$ ), the chain condenses fairly quickly into a single globule, and clusters of colors emerge and coarsen. Differently colored clusters compete, and the system ultimately evolves into an epigenetically coherent globular phase. This is markedly different from the case in which $\alpha<\alpha_{c}$, where no collapse or epigenetic ordering occurs. 


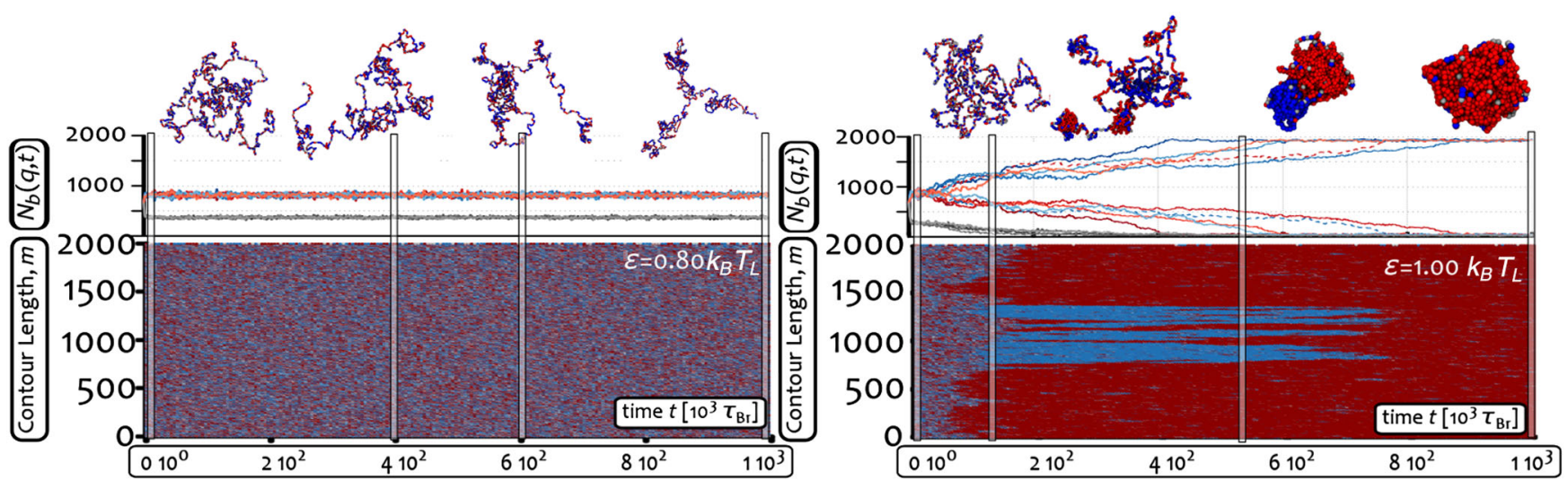

FIG. 2. The two-state model above the critical point evolves into an epigenetically coherent state via a symmetry-breaking mechanism. Top row: Typical snapshots of 3D configurations adopted by the polymers as a function of time for two choices of $\alpha=\epsilon / k_{B} T_{L}$ below and above the critical point $\alpha_{c} \simeq 0.9$ (for $M=2000$; see Ref. [54]). Middle row: Time evolution of the total number of beads of type $q$, $N_{b}(q, t)$, for four independent trajectories (the dashed one corresponds to the trajectory from which the snapshots are taken). Bottom row: Time evolution of the color of each polymer bead, viewed as a "kymograph" [57]. By tuning $\alpha>\alpha_{c}$, the whole polymer is taken over by one of the two self-attracting states via a symmetry-breaking mechanism. (see also Movies M1 and M2 in Ref. [54]).

Because the red-red and blue-blue interactions are equal, the selection of which epigenetic mark dominates is via symmetry breaking of the red $\leftrightarrow$ blue $\left(\mathbb{Z}_{2}\right)$ symmetry.

The transition between the swollen-disordered and collapsed-coherent phases bears the hallmark of a discontinuous, first-order-like transition [58,59]: For instance, we observe metastability of each of the two phases at $\alpha \simeq \alpha_{c}$ as well as marked hysteresis (see Ref. [54], Figs. S2 and S3). To better characterize the transition, we perform a set of simulations on a shorter polymer with $M=50$ beads in order to enhance sampling. We average data from 100 simulations (see Ref. [54], Fig. S4, for single trajectories), each $10^{6}$ Brownian times long, and calculate the joint probability $P\left(R_{g}, \tilde{m}\right)$ of observing a state with a given value of gyration radius, $R_{g}$, and signed "epigenetic magnetization" [32],

$$
\tilde{m} \equiv \frac{1}{M}\left(N_{b}(q=1)-N_{b}(q=2)\right) .
$$

The result (see Fig. 3 and Ref. [54], Fig. S3) shows that the single maximum expected for the swollen-disordered phase (large $R_{g}$ and small $\tilde{m}$ ) splits into two symmetric maxima corresponding to the collapsed-ordered phase (small $R_{g}$ and $\tilde{m} \simeq \pm 1$ ). More importantly, at the critical point, three maxima are clearly visible, suggesting the presence of phase coexistence (see Fig. 3 and Ref. [54], Figs. S2 and S3).

The existence of a first-order-like transition in this model provides a marked difference between our model and previous ones, which approximated the epigenetic (recoloring) dynamics as a one-dimensional process, where nucleosome recruitment was regulated by choosing an ad hoc long-range interaction [25,32]. These effectively 1D models display either a second-order transition $[25,60,61]$ or a first-order transition, but only in the mean-field ("all against all") case [32]. In our model, the first-order nature of the transition critically requires the coupling between the 3D polymer collapse and the 1D epigenetic dynamics - in this sense, the underlying physics is similar to that of magnetic polymers [62].

The dynamical feedback between chromatin folding and epigenetic recoloring can be appreciated by looking at Movies M1 and M2 in Ref. [54], where it can be seen that local epigenetic fluctuations trigger local chromatin compaction. Movies M1 and M2 also show that the dynamics of the transition from the swollen to globular phase is, to some extent, similar to that experienced by a homopolymer in poor solvent conditions [63-70], namely, a formation

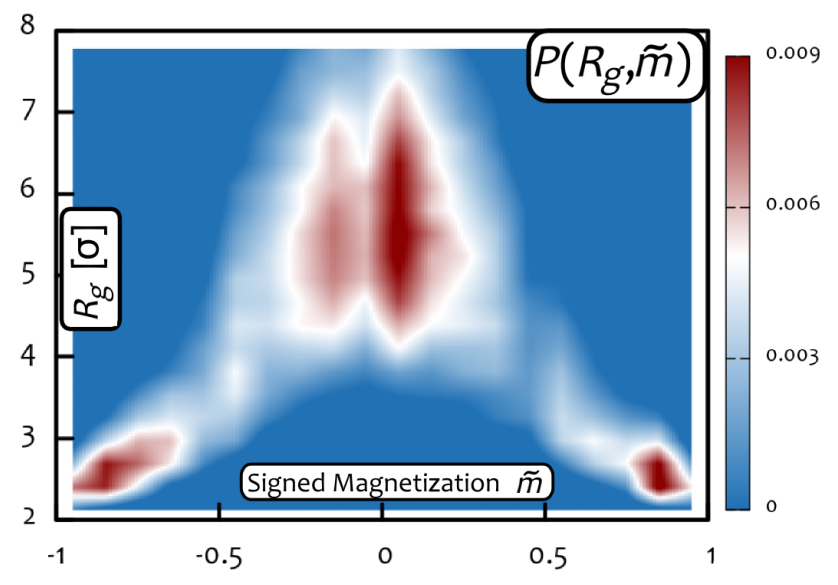

FIG. 3. The two-state model displays a discontinuous transition at the critical point marked by coexistence. The plot shows the joint probability $P\left(R_{g}, \tilde{m}\right)$ for a chain of $M=50$ beads, obtained from 100 independent simulations of duration $10^{6} \tau_{\mathrm{Br}}$ each (1000 recoloring steps) at $\alpha=1.15$ (the critical point for $M=50$ ). Single trajectories are shown in Ref. [54]. One can readily appreciate that the system displays coexistence at the critical point, therefore suggesting it is a discontinuous, first-order-like transition [see Ref. [54], Fig. S3 for plots of $P\left(R_{g}, \tilde{m}\right)$ at other values of $\alpha$ ]. 
of small compact clusters along the chain (pearls) that eventually coalesce into a single globule. Unlike the homopolymer case, however, the pearls may be differently colored, giving rise, at intermediate or late times, to frustrated dynamics, where two or more globules of different colors compete through strong surface tension effects. When several globules are present, we observe cases in which two or more pearls of the same color, which are distant along the chain but close in 3D, merge by forming long-ranged loops (see snapshots in Fig. 2, contact maps in Movies M1 and M2 in Ref. [54]).

Finally, we stress that a first-order-like transition in this system is important for biological applications since it naturally provides a framework within which epigenetic states can be established and maintained in the presence of external fluctuations. In particular, it is well known that when a gene is switched off, for instance, after development, it can very rarely be reactivated following further cellular division. This is an example of epigenetic memory, which is naturally explained within our model (as there is hysteresis). At the same time, two cell lines might display different patterns of active and inactive genes, therefore providing a clear example of epigenetic bistability, which is also recovered within this model, due to the red-blue symmetry breaking. All this strongly suggests that the features characterizing the abovementioned "epigenetic switches" may be inherited from an effective first-order-like transition driven by the coupling between epigenetic dynamics and chromatin folding such as the one displayed by the model presented here.

\section{B. Deep quenches into the collapsed phase leads to a "topological freezing" that slows down epigenetic dynamics}

An intriguing feature observed in the dynamics towards the symmetry breaking is that quenching at different temperatures nontrivially affects the time scales of chromatin condensation and epigenetic evolution towards a single coherent state (see also Movie M3 in Ref. [54]). The separation between these two time scales increases with $\alpha$ (i.e., for deeper quenches), as can be readily seen in Fig 4, where we compare the time evolution of the mean-squared radius of gyration of the chain $R_{g}^{2}(t)$ and the time-dependent (absolute) epigenetic magnetization
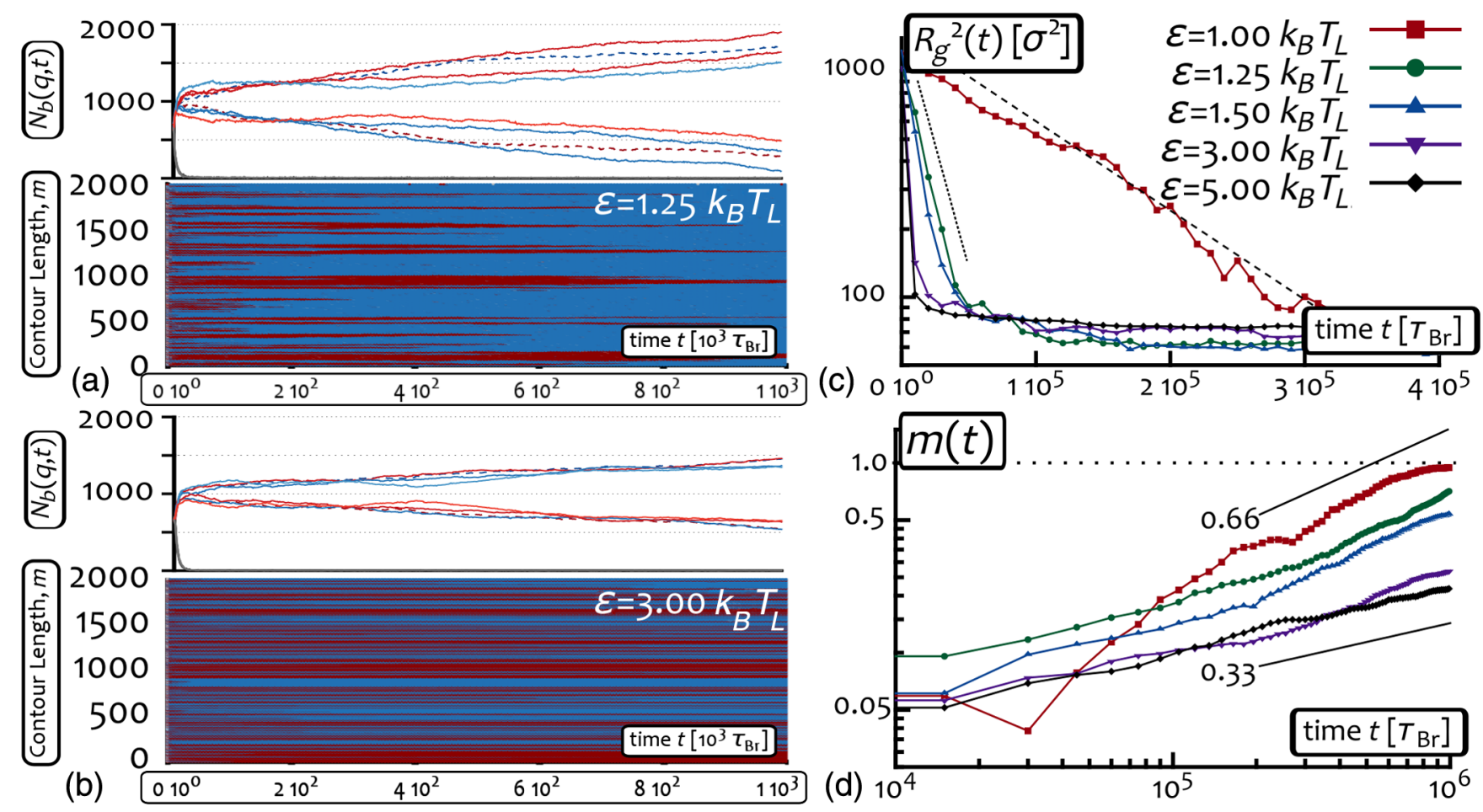

FIG. 4. Within the two-state model, epigenetic dynamics slows down with increasing $\alpha$. (a,b) These panels show the kymographs and the number of beads in state $q, N_{b}(q, t)$, for two values of $\alpha$ above the critical point $\left(\alpha_{c} \simeq 0.9\right.$ for $\left.M=2000\right)$. Counterintuitively, the symmetry breaking of the chain towards an epigenetically coherent state slows down with increasing interaction strengths (compare also with Fig. 2). (c) This panel shows the time evolution of the squared gyration radius $R_{g}^{2}$ of the polymer from the moment the collapse starts. (d) This panel (see also Movie 3 in Ref. [54]) shows the behavior of the epigenetic magnetization [defined in Eq. (3)] as a function of time. As expected, larger values of $\alpha$ therefore lead to a faster polymer collapse dynamics (faster decay of $R_{g}$ ); surprisingly, however, this is accompanied by a slower recoloring dynamics towards the epigenetically coherent state [slower growth of $m(t)$ ]. The longevity of the epigenetic domains thereby formed can be quantified by looking at the growth of the epigenetic magnetization. For $\alpha=5, m(t)$ can be extrapolated to reach, say, 0.5 at about $310^{7} \tau_{\mathrm{Br}}$, which corresponds to 5000 minutes of physical time according to our time mapping (see Sec. II). 


$$
m(t)=\frac{1}{M}\left|N_{b}(q=1, t)-N_{b}(q=2, t)\right|
$$

for different values of $\alpha$.

While $R_{g}$ decays exponentially with a time scale that decreases as $\alpha$ increases [Fig. 4(a)], the epigenetic magnetization grows as $m(t) \sim t^{\beta}$, where the dynamical exponent $\beta$ decreases from $\simeq 2 / 3$ to $\simeq 1 / 3$ as $\alpha$ increases. Note that the value $2 / 3$ has been reported in the literature as the one characterizing the coarsening of pearls in the dynamics of homopolymer collapse [65]. The fact that, in our model, this exponent is obtained for low values of $\alpha$ suggests that, in this regime, the time scales of polymer collapse and epigenetic coarsening are similar. In this case, we expect $m(t)$ to scale with the size of the largest pearl in the polymer, whose color is the most likely to be selected for the final domain-i.e., the dynamics is essentially determined by the homopolymer case. Our data are instead consistent with an apparent exponent that is smaller than $2 / 3$ for larger $\alpha$, signaling a slower epigenetic dynamics.

The interesting finding that a fast-collapse transition gives rise to a slowing down of the recoloring dynamics can be understood in terms of the evolution of the network of intrachain contacts. This can be monitored by defining the interaction matrix

$$
P_{a b}(t)= \begin{cases}1 & \text { if } d_{a b}(t)<R_{i} \\ 0 & \text { otherwise }\end{cases}
$$

where $a, b=1, \ldots, M$ denote two monomers, and $d_{a b}(t)=\left|\boldsymbol{r}_{a}(t)-\boldsymbol{r}_{b}(t)\right|$. From the interaction matrix, we can readily obtain useful information on the network structure, such as the average number of neighbors per bead,

$$
N_{n}(t)=\frac{1}{M} \sum_{a \neq b} P_{a b}(t)
$$

or the average "spanning distance," which quantifies whether the network is short or long ranged (see Ref. [54] for details). The contact probability between beads $a$ and $b$ can also be simply computed, as the time average of $P_{a b}(t)$.

As expected, for larger values of $\alpha, N_{n}(t)$ saturates to a maximum value (see Ref. [54], Fig. S9). On the other hand, and more importantly, for higher values of the interaction strength $\alpha$, a dramatic change in the spanning distance is observed. This effect is captured well by plotting a network representation of the monomer-monomer contacts, as reported in Fig. 5 (see Ref. [54], Figs. S6-S9, for a more quantitative analysis). This figure shows that, at large $\alpha$, there is a depletion of the number of edges connecting distant monomers along the chain, while short-ranged contacts are enhanced (see caption of Fig. 5 for details; see also contact maps in Ref. [54], Fig. S5). Note that this finding is consistent with the fractal, or crumpled, globule conjecture $[46,71,72]$, for which a globule obtained by a fast-collapse dynamics is rich in local contacts and poor in nonlocal ones. However, the present system represents a novel instance of an "annealed" collapsing globule, whose segments are dynamically recolored as it folds.

Finally, in order to characterize the change in the kinetics of the network, we quantify the "mobility" of the contacts,

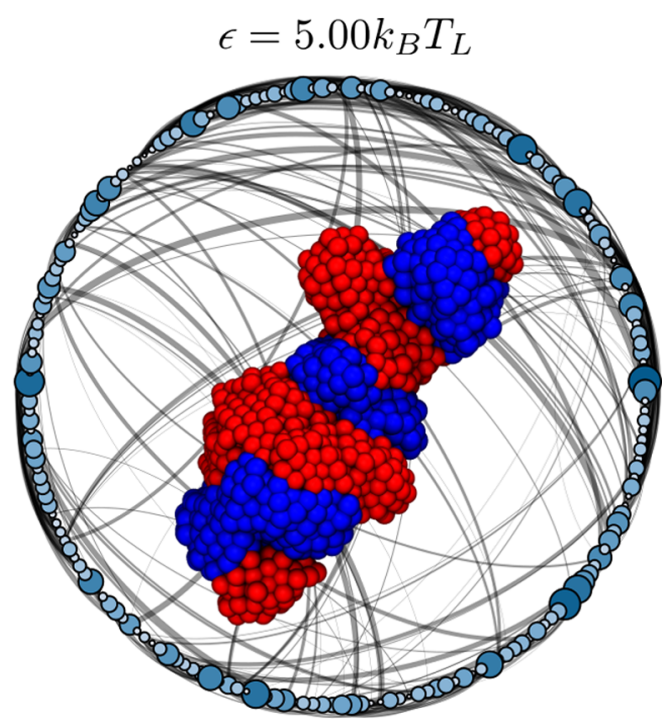

FIG. 5. The network of interactions is short-ranged for fast-collapsing coils. We show a snapshot of the network of bead-bead contacts taken at $t=10^{6} \tau_{\mathrm{Br}}$ for two simulations with (left diagram) $\epsilon=1 k_{B} T_{L}$ and (right diagram) $\epsilon=5 k_{B} T_{L}$. For clarity of visualization, each node of the network coarse grains 10 beads along the chain. The node size and color intensity encode the number of interactions within the coarse-grained monomers. Edges are only drawn between nodes that contain interacting monomers, and their thickness is proportional to the (normalized) number of contacts. To improve the visualization, only edges corresponding to contact probabilities between monomers in the top 30\% are displayed. Snapshots of the respective 3D conformations are also shown. It is important to notice that higher values of $\alpha$ lead to short-ranged networks, which translates into fewer edges but larger nodes in this coarse-grained representation. 
or the "neighbor exchange rate," following polymer collapse. We therefore compute

$$
\kappa_{n}(t)=\frac{1}{M} \sum_{a \neq b}\left[1-\delta\left(P_{a b}(t), P_{a b}(t-\Delta t)\right)\right],
$$

where $\Delta t=10^{3} \tau_{\mathrm{Br}}=\tau_{\mathrm{Rec}}$ is the gap between two measurements. We find that above $\alpha=3$, the time-averaged value of the neighbor exchange rate, normalized by the average number of neighbors, $\left\langle\kappa_{n}\right\rangle /\left\langle N_{n}\right\rangle$, sharply drops from values near unity, indicative of mobile rearranging networks, to values close to zero, signaling a frozen network or contacts (see Ref. [54], Fig. S10).

The "topological freezing" (see also Movie M3 in Ref. [54]) due to fast folding is also partially reflected by the strongly aspherical shapes taken by the collapsed coils in the large $\alpha$ regime (see snapshots in Figs. 2 and 5).

The emerging scenario is therefore markedly different from the one suggested in models for epigenetic dynamics with long-range $[25,60,61]$ or mean-field interactions [32], where any two beads in the chain would have a finite interaction probability. Instead, in our case, this is only a valid approximation at small $\alpha$; however, at large $\alpha$, a given bead interacts with only a subset of other beads (see Fig. S6), and it is only by averaging over different trajectories and beads that we get the power-law decay of the contact probability assumed in those studies (see Fig. S7). This observation is, once again, intimately related to the fact that we are explicitly taking into account the $3 \mathrm{D}$ folding together with the epigenetic dynamics.

In this section, we have therefore shown that considering large interaction strengths between the self-attracting marks (e.g., via strongly binding "readers") leads to the formation of long-lived and short-ranged domains (see Figs. 4 and 5 and contact maps in Fig. S5); while these features might be akin to the ones inferred from experimental contact maps (Hi-C) [45], both the network of interactions and the epigenetic dynamics appear to be glassy and frozen (Figs. 4 and S6-S10) on the time scales of our simulations (about 2.5-3 hours of physical time).

\section{Forcing the passage through the "unmarked" state triggers ultrasensitive kinetic response while retaining a first-order-like transition}

Up until now, our model has been based on a simple rule for the epigenetic dynamics, where each state can be transformed into any other state. In general, a specific biochemical pathway might be required to change an epigenetic mark $[1,25]$. Often, a nucleosome with a specific epigenetic mark (corresponding to, say, the "blue" state) can be converted into another state (say, the "red" one) only after the first mark has been removed. This two-step rewriting mechanism can be described by considering a "neutral" or "intermediate" state (IS) through which any nucleosome has to transit before changing its epigenetic state (say, from blue to red) $[25,27,30]$. Previous studies, based on mean-field or ad hoc power-law interaction rules for the recruitment of epigenetic marks, have shown that the presence of such an intermediate unmarked state can enhance bistability and create a long-lived MMS, in which all epigenetic states coexist in the same system [30].

Differently from the simulations reported in the previous sections, where we never observed a long-lived mixed state as the red or blue beads rapidly took over the "grey" beads, in this case, we do observe that the mixed state is metastable for a range of $\alpha \gtrsim \alpha_{c}$. The observed MMS has a characteristic lifetime much longer than the one observed for the disordered state in the two-state model when $\alpha \gtrsim \alpha_{c}$ (see Ref. [54], Fig. S12). The observed MMS is reminiscent of the one found in Ref. [30], although a difference is the absence of large-ordered domains in our case.

A typical example of a MMS is reported in the early times of Fig. 6: One can see that it is characterized by a swollen coil with no sign of epigenetic domains, and all three states coexist in the same configuration. To quantify the metastability of the mixed state, we performed 30 independent simulations and found that for $\alpha=1$ the MMS survives with probability $50 \%$ after $10^{6}$ Brownian times. By analyzing the survival probability of the MMS as a function of time (see Ref. [54], Fig. S12), we further quantified its characteristic decay time (again at $\alpha=1$ ) as $1.310^{6} \tau_{\mathrm{Br}}$, corresponding to about 3 hours in physical time according to our mapping. In contrast, we note that for $\alpha \geq 1.25$, the MMS state is unstable and never observed.

In order to study the stability of the MMS against external agents, we perturb the system by manually recoloring (in a coherent fashion) a localized fraction (10\%) of beads along the chain. From Fig. 6, one can see that, after the perturbation (performed at $t=0$ ), the chain forms a nucleation site around the artificially recolored region that eventually grows as an epigenetically coherent globule. The spreading of the local epigenetic domain throughout the whole chain can be followed from the kymograph in Fig. 6; it appears that the spreading is approximately linear until the winning mark (here in red) takes over the whole chain. The spreading may be linear because the nucleation occurs along an epigenetically disordered swollen chain, so the mark cannot easily jump long distances along the polymer because of the steep decay for long-range contacts in the swollen phase (see also Ref. [54], Movie M4 and contact maps in Fig. S11). (Note that the argument for linear spreading also applies to spontaneous nucleation, triggered by a fluctuation rather than by an external perturbation, see Ref. [54].) The spreading speed can be estimated from the "wake" left in the kymograph: It takes $0.410^{6}$ Brownian times (about 1 hour of real time) to cover $6 \mathrm{Mbp}$.

It is remarkable that, even if the spreading remained linear for a longer polymer, this speed would suffice to spread a mark through a whole chromosome. For instance, the $\mathrm{X}$ chromosome (123 Mbp) could be recolored within one cell cycle $(24 \mathrm{~h})$. All this suggests that the model presented 


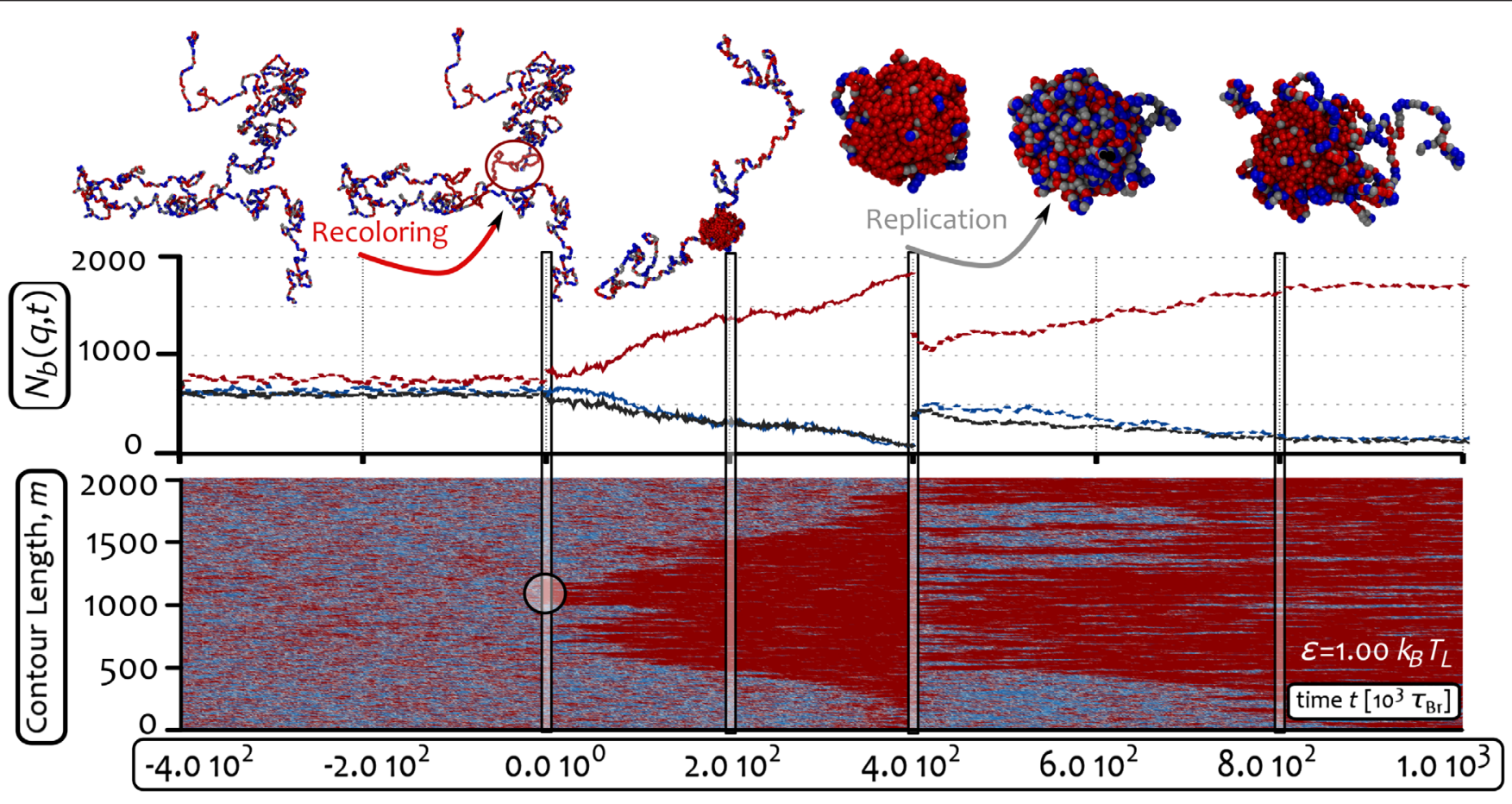

FIG. 6. The two-state-with-intermediate-state model displays ultrasensitive response to external signals such as replication or chromosome inactivation. We show the time evolution of the system starting from a mixed metastable state (MMS) and for $\epsilon=k_{B} T_{L}$. At $t=0$, a localized perturbation of the MMS is externally imposed by recoloring a segment of 200 beads (10\% of the polymer length). This perturbation triggers the collapse of the whole chain into an epigenetically coherent state that is reached within about $410^{5}$ Brownian times. At $t=410^{5} \tau_{\mathrm{Br}}$, we next simulate semiconservative replication of the collapsed chromatin fiber. This is achieved by assigning a random color to $50 \%$ of the beads all along the polymer. Following this extensive (i.e., nonlocal) color perturbation, the polymer returns to the epigenetically ordered phase. These results show that the epigenetically coherent phase is robust and stable with respect to extensive perturbations, in stark contrast to the much more sensitive MMS. Movie M4 in Ref. [54] shows the whole dynamics. Contact maps are shown in Ref. [54], Fig. S11.

in this section may thus be relevant for the fascinating $\mathrm{X}$-chromosome inactivation in embryonic mammalian cells [9] and, in more general terms, for the spreading of inactive heterochromatin along chromosomes [29].

It is also worth stressing that, in practice, for an in vivo chromatin fiber, this local coherent recoloring perturbation might be due to an increase in local concentration of a given writer (or of a reader-writer pair): Our results therefore show that a localized perturbation can trigger an extensive epigenetic response, or epigenetic switch, that might affect a large chromatin region or even an entire chromosome.

To test the stability of the coherent globular state following the symmetry breaking, we perform an extensive random recoloring of the polymer, where one of the three possible states is randomly assigned to $50 \%$ of the beads. This perturbation is chosen because it qualitatively mimics [73] how epigenetic marks may be semiconservatively passed on during DNA replication [25,27,74].

After this instantaneous extensive random recoloring (performed at $t=410^{5} \tau_{\mathrm{Br}}$ in Fig. 6), we observe that the model chromatin returns to the same ordered state, suggesting that the epigenetically coherent state, once selected, is robust to even extensive perturbations such as semiconservative replication events (see also Movie M4 in Ref. [54]).
The largely asymmetric response of the system against external perturbations, which has been shown to depend on its instantaneous state, is known as "ultrasensitivity" [26]. We have therefore shown that forcing the passage through the unmarked state triggers ultrasensitivity while retaining the discontinuous nature of the transition already captured by the simpler two-state model.

From a physics perspective, the results reported in this section and encapsulated in Fig. 6 are of interest because they show that the presence of the intermediate state does not affect the robustness of the steady states or the nature of the first-order-like transition; therefore, the previously discussed main epigenetic features of our model, memory and bistability, are maintained.

Another important remark is that ultrasensitivity is a highly desirable feature in epigenetic switches and during development. A striking example of this feature is the previously mentioned X-chromosome inactivation in mammalian female embryonic stem cells. While the selection of the chromosome copy to inactivate is stochastic at the embryonic stage, it is important to note that the choice is then epigenetically inherited in committed daughter cells [6]. Thus, in terms of the model presented here, one may imagine that a small and localized perturbation in the reading-writing 
machinery may be able to trigger an epigenetic response that drives a whole chromosome from a mixed metastable state to an inactive heterochromatic state within one cell cycle (e.g., an "all-red" state in terms of Fig. 6). When the genetic material is then replicated, we can imagine an extensive epigenetic fluctuation taking place on the whole chromosome. In turn, this extensive (global) perturbation decays over time, therefore leading to the same red heterochromatic stable state and ensuring the inheritance of the epigenetic silencing.

\section{Nonequilibrium recoloring dynamics creates a 3D organization resembling topologically associating domains}

In the previous sections, we have considered the case in which the epigenetic read-write mechanism and the chromatin folding are governed by transition rules between different microstates that obey detailed balance and that can be described in terms of an effective free energy. This is certainly a simplification because the epigenetic writing is, in general, a nonthermal, out-of-equilibrium process, which entails biochemical enzymatic reactions with chromatin remodeling and ATP consumption [1]. Thus, it is important to see what the impact is of breaking detailed balance in the dynamics of our model.

We address this point by considering a recoloring temperature $T_{\mathrm{Rec}}$ that differs from the polymer dynamics temperature $T_{L}$. When $T_{\mathrm{Rec}} \neq T_{L}$, one can readily show, through the Kolmogorov criterion, that detailed balance is violated, as there is a net probability flux along a closed loop through some of the possible states of the system (see Ref. [54]). In this case, a systematic scan of the parameter space is computationally highly demanding and outside the scope of the current work. Here, we focus on a specific case where the recoloring temperature is very low and fixed to $T_{\mathrm{Rec}}=0.1 \epsilon / k_{B} T$, while we vary $T_{L}$ : This case allows us to highlight some key qualitative differences in the behavior of the system, which are due to the nonequilibrium epigenetic dynamics. In what follows, we first discuss some expectations based on some general arguments and then present results from computer simulations.

First, imagine that the Langevin temperature $T_{L} \rightarrow \infty$. In this limit, we expect the polymer to be in the swollen disordered phase, whatever the value of $T_{\mathrm{Rec}}$ (no matter how low, as long as it is greater than zero). This is because a swollen self-avoiding walk is characterized by an intrachain contact probability scaling as

$$
P_{c}(m) \sim m^{-c}
$$

with $c=(d+\theta) \nu>2[75,76]$. This value implies that the interactions are too short-ranged to trigger a phase transition in the epigenetic state, at least within the Ising-like models considered in Ref. [60].

Consider then what happens as $T_{L}$ decreases. An important length scale characterizing order in our system is the epigenetic correlation length, which quantifies the size of the epigenetic domains along the chain. This length scale $\xi$ can be defined through the exponential decay of the epigenetic correlation function (see Ref. [54]). A second important length scale is the blob size. In particular, a homopolymer at temperature $T_{L}>\Theta$, where $\Theta$ denotes the collapse temperature, can be seen as a collection of transient de Gennes blobs with typical size [63]

$$
m^{*} \sim\left[\left(T_{L}-\Theta\right) / \Theta\right]^{-2} .
$$

Now, as $T_{L}$ decreases, remaining larger than $\Theta$, the size of the transient de Gennes blobs, $m^{*}$, increases. However, these will normally appear randomly along the chain and diffuse over the duration of the simulation to leave no detectable domain in contact maps. If, on the other hand, $\xi \sim m^{*}$, we expect states with one blob per epigenetic domain to be favored, as the epigenetic recoloring and chromatin folding would be maximally coupled. As a consequence, we may expect the resulting recoloring dynamics to slow down significantly: In this condition, chromatin domains may therefore form and be long-lived. Finally, the last regime to consider is when $T_{L}$ is small enough: In this case, we expect collapse into an epigenetically coherent globule, similarly to the results from previous sections.

To test these expectations, we now discuss computer simulations of the two-state model, where we varied $T_{L}$ while keeping $T_{\operatorname{Rec}}=0.1 \epsilon / k_{B}$. By starting from a swollen disordered polymer (which, as previously mentioned, is expected to be stable for $T_{L} \rightarrow \infty$ ), at high enough $T_{L}$, we find swollen polymers that do not form domains in the simulated contact map (see Ref. [54], this phase is also discussed more below). For lower $T_{L}$, we reach the temperature range that allows for transient blob formation. These are indeed stabilized by the existence of distinct epigenetic domains that appear at the beginning of the simulation; examples of this regime are reported in Fig. 7 and in Ref. [54] (Fig. S15).

This regime is the most interesting as the chromatin fiber displays a multipearl structure, reminiscent of the TADs found in Hi-C maps [45]. These TADs lead to a "blocklike" appearance of the contact map (see Fig. 7 and Ref. [77]), not unlike the ones reported in the literature [17,50,78]. Figure 7 also shows the number of beads in state $q$, $N_{b}(q, t)$, along with the kymograph tracking the system for $510^{6} \tau_{\mathrm{Br}}$ time steps (corresponding to about 14 hours of physical time, according to our mapping). These results show that the boundaries between domains, once established, are long-lived, as several are retained throughout the simulation. This figure should be compared and contrasted with Figs. 2 and 4, where the kymographs show either quickly disappearing domains or long-lived ones that are very small when the dynamics is glassy. In both cases, the $N_{b}(q, t)$ curves show that the system breaks the red-blue symmetry and the magnetization is diverging. Here, 

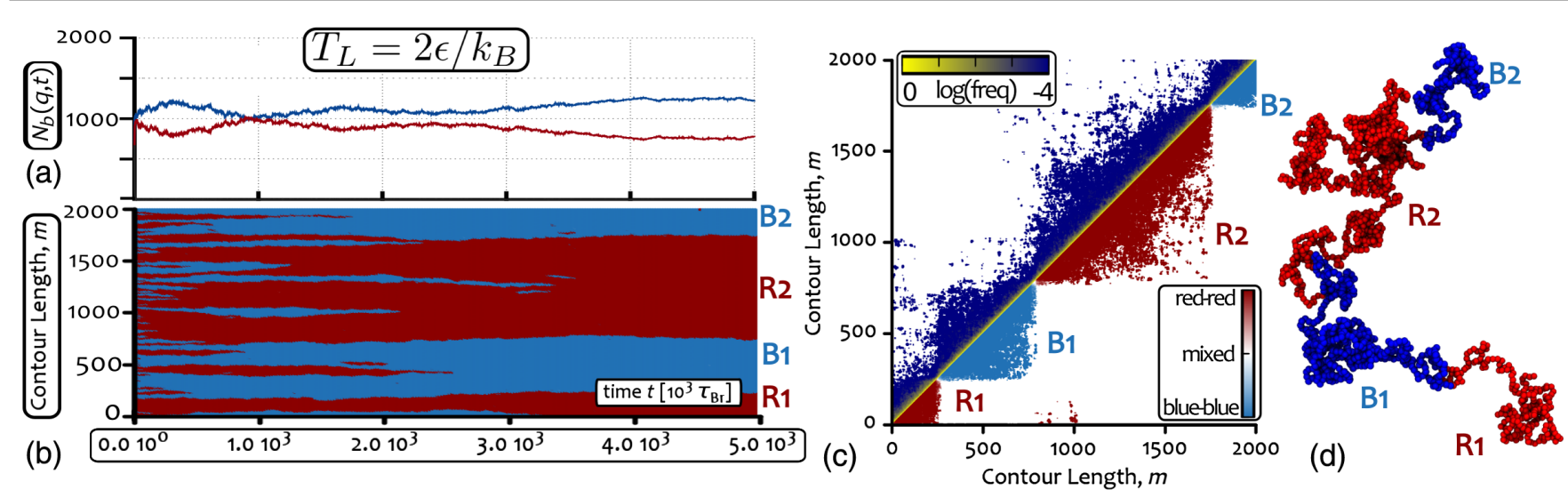

FIG. 7. Breaking detailed balance leads to the formation of TAD-like structures. Simulations correspond to $M=2000$, $T_{\text {Rec }}=0.1 \epsilon / k_{B}, T_{L}=2 \epsilon / k_{B}$ (i.e., $\alpha=\epsilon / k_{B} T_{L}=0.5$, see Ref. [54] for other cases). (a) Plot of the number of red (and blue) colored beads $N_{b}(q, t)$ as a function of time. Notice that these curves do not seem to diverge within the simulation runtime, opposite to the ones reported in the previous sections. (b) The kymograph of the system showing the presence of long-lived boundaries between distinct epigenetic domains. (c) A contact map averaged over the last $210^{5}$ Brownian times: The upper half shows the contact probability between beads; the lower half is color-coded to separately show the probability of red-red, blue-blue, and mixed contacts. (d) A snapshot of the 3D configuration. The visible TAD-like structures in the snapshot and in the contact map are enumerated as in the kymograph, to ease comparison. Note that the TAD-like structures are long-lived but metastable, while they coarsen on very long time scales. More details are given in the text and Ref. [54], and other values of $T_{L}$ are given in Figs. S14 and S15, as well as different initial conditions in Fig. S16. See also Movies.

instead, $N_{b}(q, t)$ appears to change much more slowly (or is kinetically arrested).

While the TAD-like structure observed at intermediate $T_{L}$ is long-lived, it might only be metastable, as by choosing a swollen but ordered (homopolymer) initial condition, we find that, surprisingly, no domains appear, and the polymer remains homogeneously colored throughout the simulation without collapsing into a globule. This is a signature of the existence of a swollen but epigenetically ordered phase. We recall that, remarkably, this phase cannot ever be found in the equilibrium limit of the model, $T_{L}=T_{\mathrm{Rec}}$. This new swollen and ordered regime may be due to the fact that, when $T_{L}$ decreases, the effective contact exponent will no longer be the one for self-avoiding polymers $(c>2)$, but it may be effectively closer to the one for ideal $(c=3 / 2)$ or collapsed polymers $(c=1)$, both of which allow for long-range interactions between epigenetic segments, possibly triggering epigenetic ordering (see Ref. [54], Fig. S16, and Ref. [79]).

Finally, by lowering $T_{L}$ further, below the theta point for a homopolymer $\left(T_{L} \simeq 1.8 \epsilon / k_{B}\right.$; see Ref. [54], Fig. S13), one achieves the point where the polymer collapses into a single epigenetically ordered globule (see Ref. [54], Figs. S15 and S16).

In this section, we have therefore shown that nonequilibrium epigenetic dynamics creates new features in the time evolution and steady-state behavior of the system, and may be important to understand the biophysics of TAD establishment and maintenance. Besides this, we should also mention that the domains emerging in the presented model appear randomly along the chain (i.e., no two simulations display the same epigenetic pattern); this is symptomatic of the fact that, for simplicity, our model does not consider structural and insulator elements such as the CCCTCbinding factor (CTCF), promoters, nuclear lamins, or other architectural [1] and "bookmarking" [80] proteins that may be crucial for the de novo establishment of epigenetic domains. Nonetheless, our model strongly suggests that nonequilibrium processes can play a key role in shaping the organization of chromosomes. While it has been conjectured for some time that genome regulation entails highly out-ofequilibrium processes, here we have reported a concrete instance in which breaking detailed balance naturally creates a pathway for generating a chromatin organization resembling the one observed in vivo chromosomes.

\section{DISCUSSION AND CONCLUSIONS}

In this work, we have studied a 3D polymer model with epigenetic recoloring, which explicitly takes into account the coupling between the 3D folding dynamics of a semiflexible chromatin fiber and the 1D epigenetic spreading. Supported by several experimental findings and well-established models [1,17], we assume self-attractive interactions between chromatin segments bearing the same epigenetic mark, but not between unmarked or differently marked segments. We also assume a positive feedback between readers (binding proteins aiding the folding) and writers (histone-modifying enzymes performing the recoloring), which is supported by experimental findings and 1D models [25,26,29,39,44,81].

One important novel element of the presented model is that the underlying epigenetic landscape is dynamic, while most of the previous works studying the 3D organization of 
chromatin relied on a fixed, or static, epigenetic landscape $[17,20-23,50,82]$. The dynamic nature of the epigenetic modifications is crucial to investigate the de novo selforganized emergence of epigenetically coherent domains, which is of broad relevance in development and after cell division [39].

In particular, the model presented here is able, for the first time to our knowledge, to couple the dynamics underlying epigenetic landscape to the motion of the chromatin in 3D. Furthermore, the synergy between the folding of chromatin and the spreading of histone modifications may be a crucial aspect of nuclear organization, as these two processes are very likely to occur on similar time scales. From a biological perspective, one may indeed argue that the formation of local TADs in a cell requires at least several minutes [1], while the establishment of higher-order, nonlocal contacts is even slower [82]; at the same time, histone modifications, such as acetylation or methylation, occur through enzymatic reactions whose rate is of the order of inverse seconds or minutes [39,83]. For instance, active epigenetic marks are deposited by a traveling polymerase during the nearly 10 minutes over which it transcribes an average human gene of $10 \mathrm{kbp}$ [84]. Similar considerations apply to our work as well: While the microscopic recoloring dynamics takes place over time scales of about $10^{3} \tau_{\mathrm{Br}}-10 \mathrm{~s}$, the spreading of a coherent mark (e.g., see kymographs in Figs. 2, 4, 6, and 7) may occur on time scales ranging from 5 $10^{5} \tau_{\mathrm{Br}}$ to $510^{6} \tau_{\mathrm{Br}}$, which are 5-50 times larger than the polymer reorientation time (about $10^{5} \tau_{\mathrm{Br}}$; see Ref. [54]).

Furthermore, there are examples of biological phenomena in vivo, which point to the importance of the feedback between 3D chromatin and epigenetic dynamics. A clear example is the inactivation of an active and "open" [1] chromatin region which is turned into heterochromatin. In this case, the associated methylation marks favor chromatin self-attractive interactions [84], and these, in turn, drive the formation of a condensed structure [1,39] whose inner core might be difficult to reach by other freely diffusing reactivating enzymes.

Rather fitting in this picture, we highlight that one of our main results is that the coupling between conformational and epigenetic dynamics can naturally drive the transition between a swollen and epigenetically disordered phase at high temperatures and a compact and epigenetically coherent phase at low temperatures (Fig. 2), and that this transition is discontinuous, or first-order-like, in nature (Fig. 3).

While it is known that purely short-range interactions cannot drive the system into a phase transition, effective (or ad hoc) long-range interactions within an Ising-like framework can induce a (continuous) phase transition in the thermodynamic limit $[60,61]$. In our case, importantly, the transition is discontinuous (see Fig. 3), and this is intimately related to the coupling between $3 \mathrm{D}$ and $1 \mathrm{D}$ dynamics. The physics leading to a first-order-like transition is therefore reminiscent of that at work for magnetic polymers [41] and hence fundamentally different with respect to previous works, which could not address the conformation-epigenetics positive-feedback coupling.

It is especially interesting to notice that the discontinuous nature of the transition observed in this model can naturally account for bistability and hysteresis, which are both properties normally associated with epigenetic switches.

We note that the model reported here also displays a richness of physical behaviors. For instance, we intriguingly find that by increasing the strength of self-attraction, the progress towards the final globular and epigenetically coherent phase is much slower (Fig. 4); we characterize this glasslike dynamics by analyzing the network of contacts and identifying a dramatic slowing down in the exchange of neighbors alongside a depletion of nonlocal contacts (see Fig. 5). We argue that the physics underlying the emergence of a frozen network of intrachain interactions might be reminiscent of the physics of spin glasses with quenched disorder [58,72,85] (see Fig. 5 and Ref. [54], Fig. S10).

We have also shown that the nature of the transition or the long-time behavior of the system is not affected by forcing the passage through an intermediate (neutral or unmarked) state during the epigenetic writing. In contrast, this restriction in the kinetic pathway produces major effects on the dynamics. Most notably, it allows for the existence of a long-lived MMS in which all three epigenetic states coexist even above the critical point $\alpha_{c}$ observed for the simpler two-state model. This case is interesting as it displays ultrasensitivity to external perturbations: The MMS is sensitive to small local fluctuations which drive large conformational and global changes, while the epigenetically coherent states are broadly stable against major and extensive reorganization events such as semiconservative chromatin replication (Fig. 6).

Like hysteresis and bistability, ultrasensitivity is important in in vivo situations, in order to enable regulation of gene expression and ensure heritability of epigenetic marks in development. For instance, it is often the case that, during development, a localized external stimulus (e.g., changes in the concentration of a transcription factor or a morphogen) is enough to trigger commitment of a group of cells to develop into a cell type characterizing a certain tissue rather than another [1]. On the other hand, once differentiated, such cells need to display stability against intrinsic or extrinsic noise. Ultrasensitivity similar to the one we report within this framework would enable both types of responses, depending on the instantaneous chromatin state.

A further captivating example of ultrasensitive response is the previously mentioned case of the X-chromosome inactivation. Also in that case, the selection of which of the two $\mathrm{X}$ chromosomes to silence is stochastic in female mammalian embryonic stem cells: Specifically, it is suggested that a localized increase in the level of some RNA transcripts (XistRNA) can trigger heterochromatization of 
the whole chromosome, which turns into the so-called Barr body, by propagating repressive marks through recruitment of the polycomb complex PRC2 [9]. Once the inactive X copy is selected, the choice is then epigenetically inherited in daughter cells [6], which therefore suggests robustness through disruptive replication events.

Finally, we have studied the case in which the epigenetic dynamics is subject to a different stochastic noise, with respect to the 3D chromatin dynamics. This effectively "nonequilibrium" case, where detailed balance of the underlying dynamics is broken, leads to interesting and unique physical behaviors. Possibly the most pertinent is that we observe, and justify, the existence of a parameter range for which a long-lived multipearl state consisting of several globular domains coexists, at least for a time corresponding to our longest simulation time scales, which roughly compare to 14 hours of physical time (see Fig. 7 and Sec. II for the time mapping). This multipearl structure is qualitatively reminiscent of the topologically associated domains in which a chromosome folds in vivo and requires efficient epigenetic spreading in $1 \mathrm{D}$, together with the vicinity to the theta point for homopolymer collapse in 3D.

Although one of the current paradigms of chromosome biology and biophysics is that the epigenetic landscape directs 3D genome folding [16-19,22], an outstanding question is how the epigenetic landscape is established in the first place-and how this can be reset de novo after each cell division. In this respect, our results suggest that the inherent nonequilibrium (i.e., ATP-driven) nature of the epigenetic read-write mechanism can provide a pathway to enlarge the possible breadth of epigenetic patterns that can be established stochastically, with respect to thermodynamic models.

It is indeed becoming increasingly clear that ATP-driven processes are crucial to regulate chromatin organization [86,87]; nonetheless, how this is achieved remains largely obscure [84]. The work presented here provides a concrete example of how this may occur and suggests that it would be of interest to develop experimental strategies to perturb, for instance, the interaction between reading and writing machines (e.g., by targeting the recruitment between Set1/2 and RNA polymerase, or between EZH2 and PRC, etc.), in order to determine what the effect is of the positivefeedback loop on the structure of epigenetic and chromatin domains, and to what extent these require out-ofequilibrium dynamics in order to be established.

Furthermore, we envisage that the "recolorable polymer model" formalized in this work and aimed at studying the interplay between 3D chromatin folding and epigenetic dynamics, might be extended in the future to take into account more biologically detailed (although less general) cases. For instance, one may introduce RNA polymerase as a special "writer" of active marks, which can display specific interactions with chromatin, e.g., promote looping [84]. More generally, our framework can be used as a starting point for a whole family of polymer models, which can be used to understand and interpret the outcomes of experiments designed to probe the interplay between a dynamic epigenetic landscape and chromatin organization.

To conclude, the model presented in this work can therefore be thought of as a general paradigm to study 3 D chromatin dynamics coupled to an epigenetic read-write kinetics in chromosomes. All our findings strongly support the hypothesis that positive feedback is a general mechanism through which epigenetic domains, ultrasensitivity, and epigenetic switches might be established and regulated in the cell nucleus. We highlight that, within this model, the interplay between polymer conformation and epigenetics plays a major role in the nature and stability of the emerging epigenetic states, which had not previously been appreciated and we feel ought to be investigated in future experiments.

\section{ACKNOWLEDGMENTS}

We acknowledge ERC for funding (Consolidator Grant THREEDCELLPHYSICS, Ref. No. 648050). We also wish to thank A. Y. Grosberg for a stimulating discussion in Trieste. After the present paper was submitted, we learnt of a similar work performed in the group of C. Vaillant and D. Jost, which uses a different 1D model [88].

[1] B. Alberts, A. Johnson, J. Lewis, D. Morgan, and M. Raff, Molecular Biology of the Cell (Taylor \& Francis, London, 2014), p. 1464.

[2] A. V. Probst, E. Dunleavy, and G. Almouzni, Epigenetic Inheritance During the Cell Cycle, Nat. Rev. Mol. Cell Biol. 10, 192 (2009).

[3] B. D. Strahl and C. D. Allis, The Language of Covalent Histone Modifications, Nature (London) 403, 41 (2000).

[4] T. Jenuwein and C. D. Allis, Translating the Histone Code, Science 293, 1074 (2001).

[5] B. M. Turner, Cellular Memory and the Histone Code, Cell 111, 285 (2002).

[6] M. Nicodemi and A. Prisco, Symmetry-Breaking Model for X-Chromosome Inactivation, Phys. Rev. Lett. 98, 108104 (2007).

[7] P. Avner and E. Heard, X-Chromosome Inactivation: Counting, Choice and Initiation, Nat. Rev. Genet. 2, 59 (2001).

[8] H. Marks, J. C. Chow, and S. Denissov, High-Resolution Analysis of Epigenetic Changes Associated with X Inactivation, Genome Res. 19, 1361 (2009).

[9] S. F. Pinter, R. I. Sadreyev, E. Yildirim, Y. Jeon, T. K. Ohsumi, M. Borowsky, and J. T. Lee, Spreading of $X$ Chromosome Inactivation via a Hierarchy of Defined Polycomb Stations, Genome Res. 22, 1864 (2012).

[10] S. Wood and A. Loudon, Clocks for All Seasons: Unwinding the Roles and Mechanisms of Circadian and Interval Timers in the Hypothalamus and Pituitary, J. Endocrinol. 222, R39 (2014).

[11] F. Bratzel and F. Turck, Molecular Memories in the Regulation of Seasonal Flowering: From Competence to Cessation, Genome Biol. 16, 192 (2015). 
[12] A. Angel, J. Song, C. Dean, and M. Howard, A PolycombBased Switch Underlying Quantitative Epigenetic Memory, Nature (London) 476, 105 (2011).

[13] L. Hou, D. Wang, D. Chen, Y. Liu, Y. Zhang, H. Cheng, C. $\mathrm{Xu}$, N. Sun, J. McDermott, W. B. Mair, and J.-D. J. Han, A Systems Approach to Reverse Engineer Lifespan Extension by Dietary Restriction, Cell Metabol. 23, 529 (2016).

[14] C. J. Kenyon, The Genetics of Ageing, Nature (London) 464, 504 (2010).

[15] H. N. Lim and A. van Oudenaarden, A Multistep Epigenetic Switch Enables the Stable Inheritance of DNA Methylation States, Nat. Genet. 39, 269 (2007).

[16] M. Barbieri, M. Chotalia, J. Fraser, L.-M. Lavitas, J. Dostie, A. Pombo, and M. Nicodemi, Complexity of Chromatin Folding is Captured by the Strings and Binders Switch Model, Proc. Natl. Acad. Sci. U. S. A. 109, 16173 (2012).

[17] C. A. Brackley, S. Taylor, A. Papantonis, P. R. Cook, and D. Marenduzzo, Nonspecific Bridging-Induced Attraction Drives Clustering of DNA-Binding Proteins and Genome Organization, Proc. Natl. Acad. Sci. U. S. A. 110, E3605 (2013).

[18] D. Jost, P. Carrivain, G. Cavalli, and C. Vaillant, Modeling Epigenome Folding: Formation and Dynamics of Topologically Associated Chromatin Domains, Nucleic Acids Res. 42, 9553 (2014).

[19] R. Cortini, M. Barbi, B. R. Care, C. Lavelle, A. Lesne, J. Mozziconacci, and J.-M. Victor, The Physics of Epigenetics, Rev. Mod. Phys. 88, 025002 (2016).

[20] J. R. Dixon, S. Selvaraj, F. Yue, A. Kim, Y. Li, Y. Shen, M. Hu, J. S. Liu, and B. Ren, Topological Domains in Mammalian Genomes Identified by Analysis of Chromatin Interactions, Nature (London) 485, 376 (2012).

[21] T. Sexton, E. Yaffe, E. Kenigsberg, F. Bantignies, B. Leblanc, M. Hoichman, H. Parrinello, A. Tanay, and G. Cavalli, Three-Dimensional Folding and Functional Organization Principles of the Drosophila Genome, Cell 148, 458 (2012).

[22] A. N. Boettiger, B. Bintu, J. R. Moffitt, S. Wang, B. J. Beliveau, G. Fudenberg, M. Imakaev, L. A. Mirny, C.-t. Wu, and X. Zhuang, Super-Resolution Imaging Reveals Distinct Chromatin Folding for Different Epigenetic States, Nature (London) 529, 418 (2016).

[23] E. P. Nora, B. R. Lajoie, E. G. Schulz, L. Giorgetti, I. Okamoto, N. Servant, T. Piolot, N. L. van Berkum, J. Meisig, J. Sedat, J. Gribnau, E. Barillot, N. Blüthgen, J. Dekker, and E. Heard, Spatial Partitioning of the Regulatory Landscape of the X-Inactivation Centre, Nature (London) 485, 381 (2012).

[24] L. Giorgetti, R. Galupa, E. P. Nora, T. Piolot, F. Lam, J. Dekker, G. Tiana, and E. Heard, Predictive Polymer Modeling Reveals Coupled Fluctuations in Chromosome Conformation and Transcription, Cell 157, 950 (2014).

[25] I. B. Dodd, M. A. Micheelsen, K. Sneppen, and G. Thon, Theoretical Analysis of Epigenetic Cell Memory by Nucleosome Modification, Cell 129, 813 (2007).

[26] K. Sneppen, M. A. Micheelsen, and I. B. Dodd, Ultrasensitive Gene Regulation by Positive Feedback Loops in Nucleosome Modification, Mol. Syst. Biol. 4, 182 (2008).

[27] M. A. Micheelsen, N. Mitarai, K. Sneppen, and I. B. Dodd, Theory for the Stability and Regulation of Epigenetic Landscapes, Phys. Biol. 7, 026010 (2010).
[28] I. B. Dodd and K. Sneppen, Barriers and Silencers: A Theoretical Toolkit for Control and Containment of Nucleosome-Based Epigenetic States, J. Mol. Biol. 414, 624 (2011).

[29] N. A. Hathaway, O. Bell, C. Hodges, E. L. Miller, D. S. Neel, and G. R. Crabtree, Dynamics and Memory of Heterochromatin in Living Cells, Cell 149, 1447 (2012).

[30] K. Sneppen and N. Mitarai, Multistability with a Metastable Mixed State, Phys. Rev. Lett. 109, 100602 (2012).

[31] L. C. M. Anink-Groenen, T. R. Maarleveld, P. J. Verschure, and F. J. Bruggeman, Mechanistic Stochastic Model of Histone Modification Pattern Formation, Epigenet. Chromatin. 7, 30 (2014).

[32] D. Jost, Bifurcation in Epigenetics: Implications in Development, Proliferation, and Diseases, Phys. Rev. E 89, 010701(R) (2014).

[33] H. Zhang, X.-J. Tian, A. Mukhopadhyay, K. S. Kim, and J. Xing, Statistical Mechanics Model for the Dynamics of Collective Epigenetic Histone Modification, Phys. Rev. Lett. 112, 068101 (2014).

[34] X.-J. Tian, H. Zhang, J. Sannerud, and J. Xing, Achieving Diverse and Monoallelic Olfactory Receptor Selection through Dual-Objective Optimization Design, Proc. Natl. Acad. Sci. U. S. A. 113, E2889 (2016).

[35] C. A. Brackley, M. E. Cates, and D. Marenduzzo, Intracellular Facilitated Diffusion: Searchers, Crowders, and Blockers, Phys. Rev. Lett. 111, 108101 (2013).

[36] A. M. Näär, B. D. Lemon, and R. Tjian, Transcriptional Coactivator Complexes, Annu. Rev. Biochem. 70, 475 (2001).

[37] F. Erdel, K. Müller-Ott, and K. Rippe, Establishing Epigenetic Domains via Chromatin-Bound Histone Modifiers, Ann. N.Y. Acad. Sci. 1305, 29 (2013).

[38] M. C. Barnhart, P. H. J. L. Kuich, M. E. Stellfox, J. A. Ward, E. A. Bassett, B. E. Black, and D. R. Foltz, HJURP Is a CENPA Chromatin Assembly Factor Sufficient to Form a Functional de novo Kinetochore, J. Cell Biol. 194, 229 (2011).

[39] G. E. Zentner and S. Henikoff, Regulation of Nucleosome Dynamics by Histone Modifications, Nat. Struct. Mol. Biol. 20, 259 (2013).

[40] H. H. Ng, F. Robert, R. A. Young, and K. Struhl, Targeted Recruitment of Set1 Histone Methylase by Elongating Pol II Provides a Localized Mark and Memory of Recent Transcriptional Activity, Mol. Cell 11, 709 (2003).

[41] T. Garel, H. Orland, and E. Orlandini, Phase Diagram of Magnetic Polymers, Eur. Phys. J. B 12, 261 (1999).

[42] A. H. F. M. Peters, D. O'Carroll, H. Scherthan, K. Mechtler, S. Sauer, C. Schöfer, K. Weipoltshammer, M. Pagani, M. Lachner, A. Kohlmaier et al., Loss of the Suv39h Histone Methyltransferases Impairs Mammalian Heterochromatin and Genome Stability, Cell 107, 323 (2001).

[43] G. Li, R. Margueron, M. Ku, P. Chambon, B. E. Bernstein, and D. Reinberg, Jarid2 and PRC2, Partners in Regulating Gene Expression, Genes Dev. 24, 368 (2010).

[44] S. Aranda, G. Mas, and L. Di Croce, Regulation of Gene Transcription by Polycomb Proteins, Sci. Adv. 1, e1500737 (2015).

[45] E. Lieberman-Aiden, N. L. van Berkum, L. Williams, M. Imakaev, T. Ragoczy, A. Telling, I. Amit, B. R. Lajoie, P. J. Sabo, M. O. Dorschner et al., Comprehensive Mapping of Long-Range Interactions Reveals Folding Principles of the Human Genome, Science 326, 289 (2009). 
[46] L. A. Mirny, The Fractal Globule as a Model of Chromatin Architecture in the Cell, Chromosome Res. 19, 37 (2011).

[47] A. Rosa and R. Everaers, Structure and Dynamics of Interphase Chromosomes, PLoS Comput. Biol. 4, e1000153 (2008).

[48] M. Barbieri, J. Fraser, M.-L. Lavitas, M. Chotalia, J. Dostie, A. Pombo, and M. Nicodemi, A Polymer Model Explains the Complexity of Large-Scale Chromatin Folding, Nucleus 4, 267 (2013).

[49] A. L. Sanborn, S. S. P. Rao, S.-C. Huang, N. C. Durand, M. H. Huntley, A. I. Jewett, I. D. Bochkov, D. Chinnappan, A. Cutkosky, J. Li et al., Chromatin Extrusion Explains Key Features of Loop and Domain Formation in Wild-Type and Engineered Genomes, Proc. Natl. Acad. Sci. U. S. A. 112, 10995 (2015).

[50] C. A. Brackley, J. Johnson, S. Kelly, P. R. Cook, and D. Marenduzzo, Simulated Binding of Transcription Factors to Active and Inactive Regions Folds Human Chromosomes into Loops, Rosettes and Topological Domains, Nucleic Acids Res. 44, 3503 (2016).

[51] K. Kremer and G.S. Grest, Dynamics of Entangled Linear Polymer Melts: A Molecular-Dynamics Simulation, J. Chem. Phys. 92, 5057 (1990).

[52] M. Baum, F. Erdel, M. Wachsmuth, and K. Rippe, Retrieving the Intracellular Topology from Multi-Scale Protein Mobility Mapping in Living Cells, Nat. Commun. 5, 4494 (2014).

[53] G. G. Cabal, A. Genovesio, S. Rodriguez-Navarro, C. Zimmer, O. Gadal, A. Lesne, H. Buc, F. FeuerbachFournier, J.-C. Olivo-Marin, E. C. Hurt, and U. Nehrbass, SAGA Interacting Factors Confine Sub-diffusion of Transcribed Genes to the Nuclear Envelope, Nature (London) 441, 770 (2006).

[54] See Supplemental Material at http://link.aps.org/ supplemental/10.1103/PhysRevX.6.041047 for details on the simulation procedure, further analysis on data-sets and supplementary movies.

[55] We should stress at this stage that the recoloring dynamics of epigenetic marks differs from the coloring dynamics of "designable" polymers considered in Ref. [56], where a chemical irreversible patterning is applied for some time to a short polymer in order to study its protein-folded-like conformations [56]. Here, the recoloring dynamics and the folding of the chains evolve together at all times, and they affect one another dynamically.

[56] J. Genzer, P. G. Khalatur, and A. R. Khokhlov, Polymer Science: A Comprehensive Reference, 10 Volume Set (Elsevier B.V., New York, 2012), Vol. 6, pp. 689-723.

[57] D. R. Brumley, M. Polin, T. J. Pedley, R. E. Goldstein, and R. E. Goldstein, Metachronal Waves in the Flagellar Beating of Volvox and Their Hydrodynamic Origin, J. R. Soc. Interface 12, 20141358 (2015).

[58] A. Yu. Grosberg, Collapse and Intramolecular Phase Layering in a Polymer in Which Each Unit May Be in Two States, Biophysics 29, 621 (1984).

[59] E. E. Dormidontova, A. Yu. Grosberg, and A. R. Khokhlov, Intramolecular Phase Separation of a Copolymer Chain with Mobile Primary Structure, Macromol. Theory Simul. 1, 375 (1992).

[60] A. Colliva, R. Pellegrini, A. Testori, and M. Caselle, IsingModel Description of Long-Range Correlations in DNA Sequences, Phys. Rev. E 91, 052703 (2015).
[61] F. Bouchet, S. Gupta, and D. Mukamel, Thermodynamics and Dynamics of Systems with Long-Range Interactions, Physica A (Amsterdam) 389, 4389 (2010).

[62] T. Garel and H. Orland, Mean-Field Model for Protein Folding, Europhys. Lett. 6, 307 (1988).

[63] P.-G. de Gennes, Kinetics of Collapse for a Flexible Coil, J. Phys. Lett. 46, 639 (1985).

[64] Y. A. Kuznetsov, E. G. Timoshenko, and K. A. Dawson, Kinetics at the Collapse Transition of Homopolymers and Random Copolymers, J. Chem. Phys. 103, 4807 (1995).

[65] A. Byrne, P. Kiernan, D. Green, and K. A. Dawson, Kinetics of Homopolymer Collapse, J. Chem. Phys. 102, 573 (1995).

[66] L. I. Klushin, Kinetics of a Homopolymer Collapse: Beyond the Rouse-Zimm Scaling, J. Chem. Phys. 108, 7917 (1998).

[67] N. Kikuchi, A. Gent, and J. M. Yeomans, Polymer Collapse in the Presence of Hydrodynamic Interactions, Europhys. J. E 9, 63 (2002).

[68] N. Kikuchi, J. F. Ryder, C. M. Pooley, and J. M. Yeomans, Kinetics of the Polymer Collapse Transition: The Role of Hydrodynamics, Phys. Rev. E 71, 061804 (2005).

[69] S. Rǔžička, D. Quigley, and M. P. Allen, Folding Kinetics of a Polymer, Phys. Chem. Chem. Phys. 14, 6044 (2012).

[70] C. Leitold and C. Dellago, Folding Mechanism of a Polymer Chain with Short-Range Attractions, J. Chem. Phys. 141, 134901 (2014).

[71] A. Yu. Grosberg, S. K. Nechaev, and E. I. Shakhnovich, The Role of Topological Constraints in the Kinetics of Collapse of Macromolecules, J. Phys. France 49, 2095 (1988).

[72] C. D. Sfatos and E. I. Shakhnovic, Statistical Mechanics of Random Heteropolymers, Phys. Rep. 288, 77 (1997).

[73] Another strategy that we have tested is to turn $50 \%$ of the beads into inactive, grey monomers, as this may more faithfully represent what happens immediately after replications. The results are qualitatively similar to the ones discussed in the text, although we observe that the system returns to the collapsed ordered state more quickly in this case.

[74] M. B. Zerihun, C. Vaillant, and D. Jost, Effect of Replication on Epigenetic Memory and Consequences on Gene Transcription, Phys. Biol. 12, 026007 (2015).

[75] S. Redner, Distribution Functions in the Interior of Polymer Chains, J. Phys. A 13, 3525 (1980).

[76] B. Duplantier, Exact Contact Critical Exponents of a SelfAvoiding Polymer Chain in Two Dimensions, Phys. Rev. B 35, 5290 (1987).

[77] The colored contact map is computed by weighting each observed contact with the types of interacting beads $(-1,1$, or 0 for blue-blue, red-red, or mixed contacts, respectively) and by normalizing each entry by its total number of contacts. This procedure allows us to identify the epigenetic domains observed in the 3D snapshots and to demonstrate that, in this case, the model displays domains that have intraTAD contacts between the same colored beads, as shown by the roughly uniform color throughout each domain (more details are given in Ref. [54]—for a full dynamics, see also Movies M5 and M6).

[78] J. Dekker, K. Rippe, M. Dekker, and N. Kleckner, Capturing Chromosome Conformation, Science 295, 1306 (2002). 
[79] With $T_{\operatorname{Rec}}=0.1 \epsilon / k_{B}$, we cannot simulate large enough $T_{L}$ to probe the swollen disordered regime: This may signal the fact that $\xi=\infty$ at small enough nonzero $T_{\operatorname{Rec}}$, but we cannot exclude it to be a finite-size effect (if we simulate polymers with $M<\xi$ ). On the other hand, the swollen disordered phase can be easily observed at, e.g., $T_{\text {Rec }}=0.5 \epsilon / k_{B}$.

[80] K. D. Sarge and O. K. Park-Sarge, Gene Bookmarking: Keeping the Pages Open, Trends Biochem. Sci. 30, 605 (2005).

[81] K. Müller-Ott, F. Erdel, A. Matveeva, J.-P. Mallm, A. Rademacher, M. Hahn, C. Bauer, Q. Zhang, S. Kaltofen, G. Schotta, T. Höfer, and K. Rippe, Specificity, Propagation, and Memory of Pericentric Heterochromatin, Mol. Syst. Biol. 10, 746 (2014).

[82] D. Michieletto, D. Marenduzzo, and A.H. Wani, Chromosome-Wide Simulations Uncover Folding Pathway and 3D Organization of Interphase Chromosomes, arXiv: 1604.03041.
[83] T. K. Barth and A. Imhof, Fast Signals and Slow Marks: The Dynamics of Histone Modifications, Trends Biochem. Sci. 35, 618 (2010).

[84] P. R. Cook, Principles of Nuclear Structure and Function (Wiley, New York, 2001).

[85] T. Garel, H. Orland, and E. Pitard, Protein Folding and Heteropolymers, in Spin Glasses and Random Fields, edited by A. P. Young (World Scientific, Singapore, 1997), p. 57.

[86] A. Goloborodko, J. F. Marko, and L. A. Mirny, Chromosome Compaction by Active Loop Extrusion, Biophys. J. 110, 2162 (2016).

[87] A. Goloborodko, M. V. Imakaev, J. F. Marko, and L. Mirny, Compaction and Segregation of Sister Chromatids via Active Loop Extrusion, eLife 2016, e14864 (2016).

[88] N. Haddad, D. Jost, and C. Vaillant, Perspectives: Using Polymer Modeling to Understand the Formation and Function of Nuclear Compartments, Chromosome Res. (to be published). 